\title{
Treatment of Gingival Recession with Microinvasive Surgical Technology
}

\author{
Qiong Cao $\mathbb{D},{ }^{1}$ Ruohuang Lu, ${ }^{2}$ Jun Chen, ${ }^{1}$ Hao Pan, ${ }^{1}$ Hui Feng, ${ }^{1}$ Binjie Liu $\mathbb{D},{ }^{1}$ \\ and Yuehong Wang ${ }^{1}$
}

\author{
${ }^{1}$ Hunan Key Laboratory of Oral Health Research \& Hunan 3D Printing Engineering Research Center of Oral Care \& Hunan Clinical \\ Research Center of Oral Major Diseases and Oral Health \& Academician Workstation for Oral-Maxillofacial and Regenerative \\ Medicine \& Xiangya School of Stomatology, Central South University, Changsha 410008, China \\ ${ }^{2}$ Department of Stomatology, The Third Xiangya Hospital, Affiliated to Central South University, Changsha, Hunan, China
}

Correspondence should be addressed to Binjie Liu; liubinjie@188.com and Yuehong Wang; wangyuehong1999@csu.edu.cn

Received 13 March 2021; Revised 3 April 2021; Accepted 9 April 2021; Published 28 April 2021

Academic Editor: tingting Hong

Copyright (c) 2021 Qiong Cao et al. This is an open access article distributed under the Creative Commons Attribution License, which permits unrestricted use, distribution, and reproduction in any medium, provided the original work is properly cited.

Objective. The purpose of this clinical research was to evaluate the result of microinvasive surgical technology: vestibular incision subperiosteal tunnel access (VISTA) and subepithelial connective tissue graft (SCTG) in multiple gingival recession. Methods. A total of 20 patients with 25 Miller I and 30 Miller III gingival recession teeth were treated with VISTA+SCTG. The data at baseline and 12 months were assessed: probing depth (PD), clinical attachment loss (CAL), gingival recession depth (RD), gingival recession width (RW), width of keratinized tissue (WKT), and gingival biotype (GB), and percentage of root coverage (RC) and complete root coverage (CRC) were calculated. Results. The average root coverage was $1.52 \pm 0.70 \mathrm{~mm}$ in Miller I and $0.82 \pm 0.79 \mathrm{~mm}$ in Miller III. The mean root coverage rate was $99.00 \% \pm 5.00 \%$ in Miller I and $60.73 \pm 37.90 \%$ in Miller III. The width of clinical attachment loss of keratinized tissue was significantly improved. Conclusions. VISTA and SCTG are effective in the treatment of both Miller class I and III multiple gingival recessions. Gingival increment in Miller class I is better than that in III. It is the same for maxillary and mandibular teeth.

\section{Introduction}

Multiple gingival recession (GR) is a common clinical symptom after the destruction of periodontal tissues, which causes gingival apical displacement and exposure of the cementoenamel junction. It is defined as gingiva receding in successive teeth and often leads to sensitive root surface exposure, root surface caries, and wedge-shaped defects and affects the therapeutic effect of the esthetic area, particularly in those patients with gummy smile. The susceptible factors of gingival recession are biological type of thin gingiva, protrusion of teeth outside the dental arch, bad oral hygiene, wrong way of brushing teeth, and poor control of periodontitis [1-4].

Traditional surgical methods for treatment of gingival recession defects include coronally advanced flap (CAF) and laterally advanced flap (LAF) [5]. CAF+SCTG may be considered the gold standard in terms of root coverage predictability and long-term stability of outcomes [6-9]. With the development of microsurgical instruments and materials, the technique is improved to modified coronally advanced tunnel (MCAT)+SCTG after many periodontal experts completed researches $[1,2,10,11]$.

According to Miller classification, classes III and IV are supposed to have only partial root coverage, and the relevant research is scarce [12-14]. VISTA technique was designed by Zadeh [15] combined with SCTG to treat gingival recession defects, which is an effective minimally invasive and modified coronally advanced tunnel [16]. This study analyzes and compares the clinical efficacy of this technique in the treatment of multiple Miller I and III gingival recession defects.

\section{Material and Methods}

2.1. Study Design and Patient Selection. This study was approved by the institutional review board and human subject ethics board (No. 20180027) and was conducted in 




Figure 1: Miller class I gingival recessions with wedge-shaped defects, buccal view

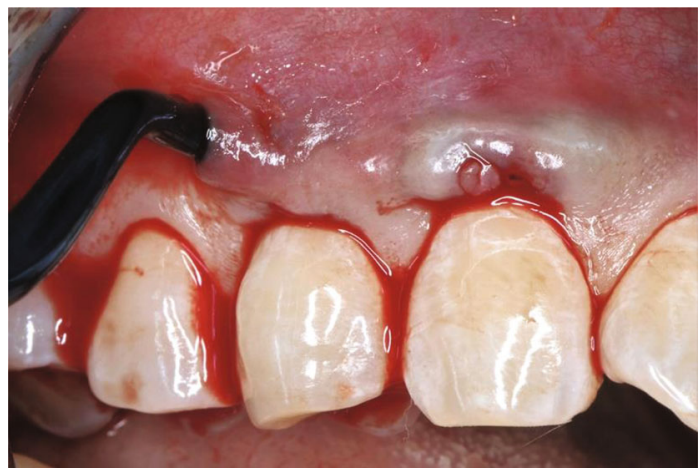

FIGURE 2: VISTA instrument prepared tunnel to allow gingival coronal advancement

accordance with the Helsinki Declaration of 1975, as revised in 2013.

Twenty patients were referred to the periodontal department of Xiangya Stomatological Hospital from August 2018 to April 2019 with a chief complaint of dentinal hypersensitivity or esthetic concern with gingival recession defects and were enrolled in the study. Some of them have slightly wedge-shaped defects, but not serious. All patients signed an informed consent form to participate.

Inclusion criteria were as follows: (1) at least two adjacent teeth with Miller class I or III gingival recession defects and (2) all patients who underwent professional periodontal initial therapy and received oral hygiene instruction with the full-mouth plaque and bleeding scores less than $20 \%$ and periodontal probing depth less than or equal to $3 \mathrm{~mm}$.

Exclusion criteria were as follows: (1) pregnant women; (2) serious systemic diseases; (3) taking drugs that interfere with healing; and (4) smokers.

2.2. Procedure. All patients completed the periodontal initial therapy including supragingival scaling, subgingival scaling, and root planning. Six to 8 weeks after these treatments, the periodontal examination showed that the inflammation is controlled to stability without bleeding. The operation was carried out on the position of gingival recession. Postoperative follow-up was conducted on time to analyze the treatment effect.

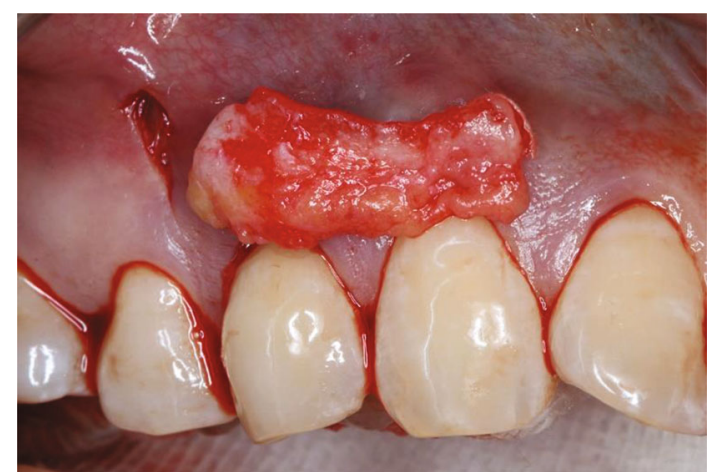

FIgURE 3: Subepithelial connective tissue graft from the palate was harvested and adapted

2.3. Clinical Measurements. Prior to surgery, the following baseline data were collected: probing depth (PD), clinical attachment loss (CAL), gingival recession depth (RD), gingival recession width (RW) at the cementoenamel junction (CEJ), width of keratinized tissue (WKT), and gingival biotype (GB). All data were recorded again at 12 months after the operation. Percentage of root coverage (RC) was calculated as (preoperative recession depth - postoperative recession depth)/preoperative recession depth $* 100 \%$. Complete root coverage (CRC) was calculated as complete root covered teeth/all teeth $* 100 \%$. All the observed indexes were examined by the same periodontal specialist who had passed the standard consistency test (Kappa value $=0.89$ ).

2.4. Surgical Procedure (Figures 1-9). The operative methods were VISTA combined with SCTG. After local infiltration anesthesia taking effect in the operation area (Figure 1), the longitudinal incision at the labial-buccal frenulum near the operation area was used as the surgical approach to separate the soft tissue from the bone surface. It was critical to elevate the tunnel region sufficiently beyond the mucogingival junction, so that the gingival flap could be fully relaxed and coronal repositioning is without tension (Figure 2). In the donor site, a horizontal incision was made on $3 \mathrm{~mm}$ below the palatal gingival margin of maxillary 4 to 6 . After the partialthickness flap was elevated, the SCTG was obtained with a thickness of $1 \sim 2 \mathrm{~mm}$. Then, it was inserted into the tunnel of the recipient area from the longitudinal incision (Figures 3 and 4). The gingival flap and graft were suspended coronally to cover the root surface beyond the CEJ of the crown, and the 5-0 nonabsorbable suture was fixed on the buccal surface of the teeth with flowing resin (Figures 5 and 6). After the operation, patients were treated with amoxicillin and $0.12 \%$ chlorhexidine gargle for one week. Stitches were removed after 7-10 days (Figure 7). All patients were recalled at 12 months (Figures 8 and 9).

2.5. Statistical Analysis. SPSS 18.0 software was used for statistical analysis. The measurement data were expressed as mean \pm standard deviation. The data before and 12 months after operation were compared by paired $t$-test. Independent sample $t$-test was used to compare the data between Miller I and III. When the $P$ value $<0.05$, the difference was considered statistically significant. 


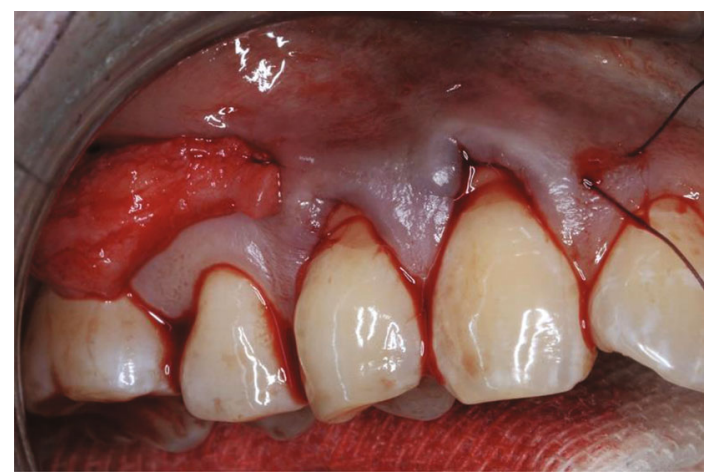

FIGURE 4: Subepithelial connective tissue graft was pulled into the tunnel by means of mattress sutures

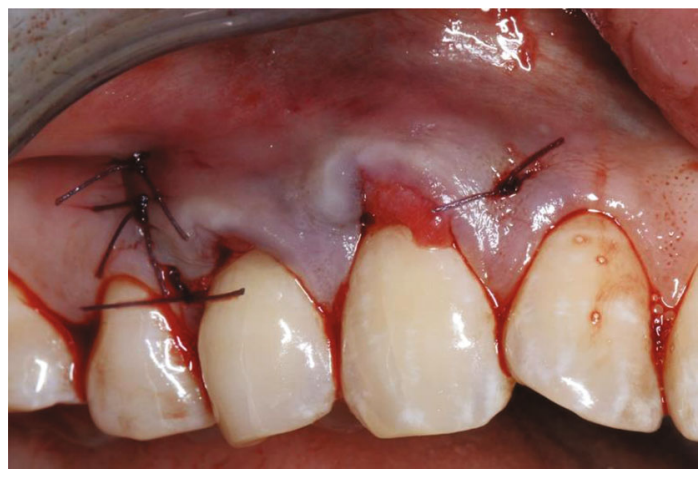

FIGURE 5: Fixation of the subepithelial connective tissue graft in the tunnel

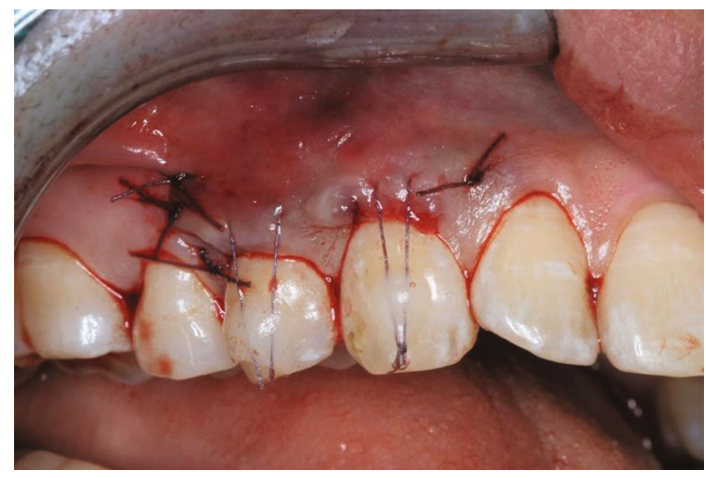

FIGURE 6: Coronally anchored bonded sutures

\section{Results}

A total of 20 patients ( 9 males and 11 females) were included, aged from 23 to 67 with an average of 35 . There were 55 teeth, including 25 Miller I and 30 Miller III gingival recession teeth as well as 21 maxillary and 34 mandibular teeth.

The depth of gingival recession before and 12 months after operation is shown in Table 1. It can be seen that the GD of Miller class I decreased from $1.54 \pm 0.71 \mathrm{~mm}$ to 0.02 $\pm 0.10 \mathrm{~mm}(P<0.05)$, and the average $\mathrm{RC}$ was $1.52 \pm 0.70$ $\mathrm{mm}$. The average RC rate was $99.00 \% \pm 5.00 \%$, and 24 of

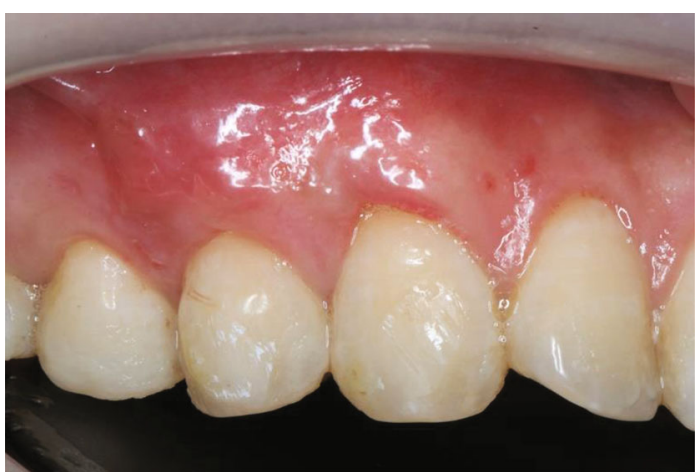

FIGURE 7: Ten-day postoperative

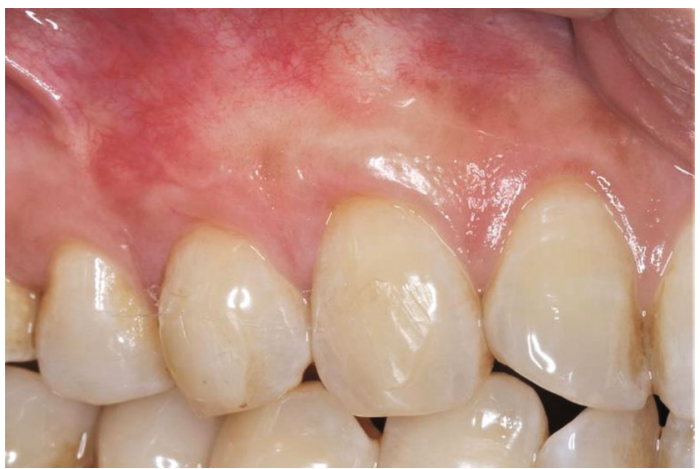

FIGURE 8: Six-month postoperative

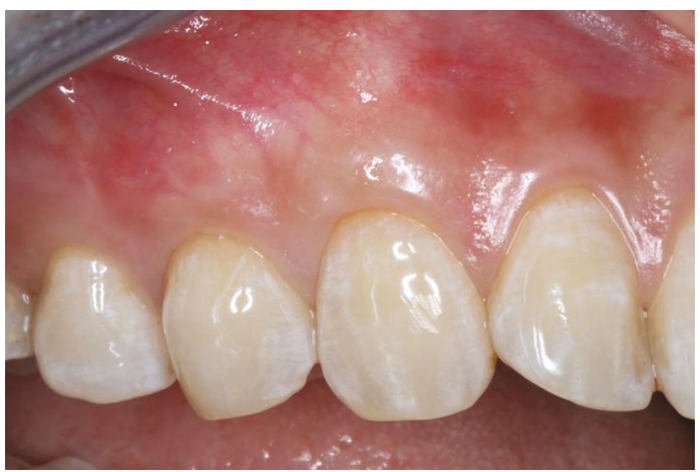

Figure 9: Twelve-month postoperative

the 25 teeth achieved CRC. That is, the CRC rate was $96.00 \%$. The GD of Miller class III decreased from $1.50 \pm$ $1.01 \mathrm{~mm}$ to $0.68 \pm 0.71 \mathrm{~mm}(P<0.05)$, and the average $\mathrm{RC}$ was $0.82 \pm 0.79 \mathrm{~mm}$. The average $\mathrm{RC}$ rate was $60.73 \% \pm$ $37.90 \%$. There was no significant difference in the PD between preoperation and postoperation; however, the width of CAL, WKT, and RW was significantly improved.

Comparing the gingival changes of Miller I and III between preoperation and postoperation, Table 2 showed that the RC including width and depth of Miller I was more than that of Miller III, as well as CAL change. But there was no significant difference in WKT. The data of maxillary and 
TABLE 1: Comparing data before and 12 months after operation.

\begin{tabular}{|c|c|c|c|c|c|}
\hline Parameter & & Baseline & 12 months & Difference & $P$ value ${ }^{\ddagger}$ \\
\hline \multirow{2}{*}{$\mathrm{RD}(\mathrm{mm})$} & Miller I & $1.54 \pm 0.71$ & $0.02 \pm 0.10$ & $1.52 \pm 0.70$ & $<0.01$ \\
\hline & Miller III & $1.50 \pm 1.01$ & $0.68 \pm 0.71$ & $0.82 \pm 0.79$ & $<0.01$ \\
\hline \multirow{2}{*}{$\mathrm{KT}(\mathrm{mm})$} & Miller I & $2.40 \pm 1.20$ & $2.86 \pm 1.03$ & $0.46 \pm 0.61$ & $<0.01$ \\
\hline & Miller III & $2.22 \pm 0.85$ & $2.77 \pm 0.72$ & $0.55 \pm 0.71$ & $<0.01$ \\
\hline \multirow{2}{*}{$\mathrm{PD}(\mathrm{mm})$} & Miller I & $1.89 \pm 0.31$ & $1.93 \pm 0.28$ & $0.04 \pm 0.15$ & 0.207 \\
\hline & Miller III & $1.84 \pm 0.26$ & $1.86 \pm 0.29$ & $0.02 \pm 0.13$ & 0.501 \\
\hline \multirow{2}{*}{$\mathrm{CAL}(\mathrm{mm})$} & Miller I & $3.10 \pm 0.58$ & $1.82 \pm 0.43$ & $1.28 \pm 0.56$ & $<0.01$ \\
\hline & Miller III & $3.23 \pm 1.14$ & $2.45 \pm 0.86$ & $0.78 \pm 0.69$ & $<0.01$ \\
\hline \multirow{2}{*}{$\mathrm{RW}(\mathrm{mm})$} & Miller I & $3.08 \pm 0.70$ & $0.12 \pm 0.60$ & $2.96 \pm 0.79$ & $<0.01$ \\
\hline & Miller III & $3.18 \pm 0.84$ & $1.85 \pm 1.61$ & $1.33 \pm 1.12$ & $<0.01$ \\
\hline
\end{tabular}

RD: gingival recession depth; KT: width of keratinized tissue; PD: probing depth; CAL: clinical attachment loss; RW: gingival recession width. Data reported as mean $\pm \mathrm{SD}$, in $\mathrm{mm}$. ${ }^{\ddagger}$ Statistically significant difference-paired $t$-test, $P<0.05$.

TABLE 2: Comparison of preoperative and 12 months postoperative gingival changes.

\begin{tabular}{|c|c|c|c|c|c|c|}
\hline \multirow{2}{*}{ Parameter } & \multicolumn{2}{|c|}{ Miller classification } & \multicolumn{2}{|c|}{ Teeth position } & \multicolumn{2}{|c|}{$P$ value ${ }^{\S}$} \\
\hline & Miller I $(n=25)$ & Miller III $(n=30)$ & Maxillary $(n=21)$ & Mandibular $(n=34)$ & Miller classification & Teeth position \\
\hline $\mathrm{RD}(\mathrm{mm})$ & $1.52 \pm 0.70$ & $0.82 \pm 0.79$ & $1.67 \pm 0.86$ & $0.81 \pm 0.62$ & $<0.01$ & $<0.01$ \\
\hline $\mathrm{KT}(\mathrm{mm})$ & $0.46 \pm 0.61$ & $0.55 \pm 0.71$ & $0.69 \pm 0.73$ & $0.40 \pm 0.60$ & 0.621 & 0.112 \\
\hline CAL (mm) & $1.28 \pm 0.56$ & $0.78 \pm 0.69$ & $1.33 \pm 0.66$ & $0.81 \pm 0.62$ & $<0.01$ & $<0.01$ \\
\hline $\mathrm{RW}(\mathrm{mm})$ & $2.96 \pm 0.79$ & $1.33 \pm 1.12$ & $2.88 \pm 1.09$ & $1.57 \pm 1.12$ & $<0.01$ & $<0.01$ \\
\hline $\mathrm{RC}(\%)$ & $99.00 \% \pm 5.00 \%$ & $60.73 \% \pm 37.90 \%$ & $94.70 \pm 13.89$ & $67.89 \pm 38.55$ & & \\
\hline CRC (\%) & $96.00 \%$ & $36.67 \%$ & $85.71 \%$ & $50 \%$ & & \\
\hline
\end{tabular}

RD: gingival recession depth change; KT: width of keratinized tissue change; CAL: clinical attachment loss change; RW: gingival recession width change; RC: percentage of root coverage $=$ gingival recession depth change divided by preoperative recession depth; CRC: complete root coverage $=$ complete root covered teeth divided by all teeth. Data reported as mean $\pm \mathrm{SD}$, in $\mathrm{mm}$. ${ }^{\S}$ Statistically significant difference-independent sample $t$-test, $P<0.05$.

mandibular teeth were also compared in Table 2. The RC (width and depth) of maxillary teeth was significantly higher than that of mandibular, as well as CAL.

\section{Discussion}

As a dentist, we used to treat oral diseases and restore missing teeth, but we have entered a new era of pink and white esthetics. It means that we have stricter standards about the appearance of the gingiva and teeth whether it is beautiful and harmonious $[17,18]$. More dentists as well as patients pay attention to the related problems caused by gingival recession. The treatment of gingival recession and the maintenance of periodontal health have become an indispensable part of general oral treatment.

The VISTA technology is invented by the professor Zadeh combined with SCTG to treat gingival recession [15]. This is a minimally invasive modified coronally advanced tunnel technique. The main purpose of this esthetic surgery is to obtain the coverage of root surface and the amount of buccal soft tissue and reduce the complications caused by surgical techniques, such as the destruction of blood supply of soft tissue flap and gingival papilla caused by traditional coronally advanced flap. Unlike the common tunnel technique, the surgical incision approach is very small, located in the vestibular sulcus. This approach can avoid gingival damage caused by repeated traction and other operations during the process away from the thinnest gingival margin of soft tissue defect, while the soft tissue is lifted and separated from the bone surface, which created a stable space for the graft and kept the gingival papilla and blood supply beneath undestroyed. Good blood supply not only is the basis of graft survival but also provides favorable conditions for the prognosis of the operation. This coronally advanced tunnel gingival tissue is suspended sutured and fixed on the tooth surface, which is the promoting factor for the relative stability of soft tissue and the formation of attachment after operation.

In the study of Santamaria et al., Miller class I and II gingival recession was treated with TUN+CTG. The average RC and the CRC rates were $77.4 \% \pm 20.4 \%$ and $28.6 \%$, respectively [19]. The coronally advanced suspended suture improved based on the tunnel technique is more beneficial to the effect of root surface coverage. Thalmair et al. reported that Miller class I and II defects were treated via a modified tunnel technique with SCTG, with an average RC rate of $93.87 \%$ and CRC rate of $74.60 \%$ [20]. In the report of Rajeswari et al., Miller Class I and II gingival recession of multiple 
teeth was treated with VISTA combined with collagen membrane and platelet-rich fibrin, and the mean RC rate was $94.17 \% \pm 8.42 \%$ at 18 months [21]. New methods had emerged to improve the effectiveness of the operation.

Miller classification has a great influence on the therapeutic effect. In our study, the average RC rate of VISTA + SCTG in Miller I and III gingival recession was $99.00 \% \pm$ $5.00 \%$ and $60.73 \% \pm 37.90 \%$, respectively. In the research results of other scholars, Yaman et al. used MCAT+SCTG to treat 9 cases of continuous Miller III gingival recession [14]. One year after the operation, the CRC rate was $50 \%$, and the average RC rate was $78 \%$. At 12 months, the interdental space filling rate was $73 \%$. Studies have shown that MCAT+SCTG may be an effective method for the treatment of multiple adjacent Miller III gingival recession, especially when there is a defect in the space between adjacent teeth. However, the success rate of Miller III root surface covering surgery may be related to the amount of tissue before surgery, such as gingival thickness, recession depth and width, and gingival tension [22]. After linear regression analysis, Velilla Esteibar et al. found that the width of the initial gingival recession was less than $3 \mathrm{~mm}$, the thickness of the graft was more than $2 \mathrm{~mm}$, the interproximal soft tissue integrity was preserved, and the interproximal bone defect was less than $3 \mathrm{~mm}$ [23]. In all these cases of Miller III gingival recession, it was possible to achieve CRC. If this phenomenon is confirmed by further research, the Miller classification could be further extended. It is assumed that the lesser the defect of the interproximal soft tissue of Miller III gingival recession and the closer it is to Miller I, the better the prognosis of the operation.

The RC rate of maxillary teeth is better than that of mandibular teeth, which is similar to the results of Aroca et al. [24]. From the perspective of anatomy and physiology, the difference in the effect of surgery between the maxillary and mandibular teeth can be explained by the different structures of the gingival papilla. That is, the maxillary gingival papilla is larger and wider, which makes its blood supply rich and provides space stability. Another explanation is that the attachment of the upper lip muscle of the mandible is lower and the depth of the vestibular sulcus is shallow, which may lead to the difficulty of coronal reduction and the decrease of RC. To my mind, the maxillary cortical plate is loose and porous with good blood supply, while the maxillary cortical plate is thick with slightly poor blood supply, which may also be the reason affecting the surgical effect.

\section{Conclusions}

The results of this study show that VISTA and SCTG are effective in the treatment of Miller class I and III continuous gingival recession. Twelve months after operation, RC was obtained in all patients with gingival recession, and the clinical root surface coverage was statistically significant. Most importantly, as the risk factors of gingival recession, thin gingival biotype has turned into a thick gingival biotype after operation. This trend reduces the risk of gingival recession. This is a kind of predictable soft tissue reconstruction and has a good esthetic effect. Further studies will provide more evidence about this operation.

\section{Data Availability}

Data sharing is not applicable. Further studies are under way.

\section{Conflicts of Interest}

The authors do not have any financial conflict of interest.

\section{Authors' Contributions}

All authors have made substantial contributions to conception and design of the study. Qiong Cao, Jun Chen, Hao Pan, and Hui Feng have been involved in data collection and data analysis. Qiong Cao, Binjie Liu, Yuehong Wang, and Ruohuang Lu have been involved in data interpretation, drafting the manuscript, and revising it critically and have given final approval of the version to be published.

\section{Acknowledgments}

This work was partially supported by the National Natural Science Foundation of China (No. 81901020); Natural Science Foundation of Hunan Province, China (No. 2020JJ4458); and Hunan Provincial Science and Technology Department clinical medical technology innovation guide project (No. S2020SFYLJS0571).

\section{References}

[1] Q. Ding, J. Cui, H. Shen et al., "Advances of nanomaterial applications in oral and maxillofacial tissue regeneration and disease treatment," Wiley Interdisciplinary Reviews: Nanomedicine and Nanobiotechnology, vol. 13, no. 2, 2021.

[2] D. Lauritano, L. Limongelli, G. Moreo, G. Favia, and F. Carinci, "Nanomaterials for periodontal tissue engineering: chitosan-based scaffolds. A systematic review," Nanomaterials, vol. 10, no. 4, p. 605, 2020.

[3] G. Zucchelli, P. Sharma, and I. Mounssif, "Esthetics in periodontics and implantology," Periodontology 2000, vol. 77, no. 1, pp. 7-18, 2018.

[4] M. Stefanini, M. Marzadori, S. Aroca, P. Felice, M. Sangiorgi, and G. Zucchelli, "Decision making in root-coverage procedures for the esthetic outcome," Periodontology 2000, vol. 77, no. 1, pp. 54-64, 2018.

[5] A. Azaripour, M. Kissinger, V. S. L. Farina et al., "Root coverage with connective tissue graft associated with coronally advanced flap or tunnel technique: a randomized, doubleblind, mono-centre clinical trial," Journal of Clinical Periodontology, vol. 43, no. 12, pp. 1142-1150, 2016.

[6] D. J.-M. Leong and H.-L. Wang, "A decision tree for soft tissue grafting," International Journal of Periodontics and Restorative Dentistry, vol. 31, no. 3, pp. 307-313, 2011.

[7] F. Cairo, M. Nieri, and U. Pagliaro, "Efficacy of periodontal plastic surgery procedures in the treatment of localized facial gingival recessions. A systematic review," Journal of Clinical Periodontology, vol. 41, pp. S44-S62, 2014.

[8] A. Sculean, R. Cosgarea, A. Stähli et al., "The modified coronally advanced tunnel combined with an enamel matrix 
derivative and subepithelial connective tissue graft for the treatment of isolated mandibular Miller class I and II gingival recessions: a report of 16 cases," Quintessence International, vol. 45, no. 10, pp. 829-835, 2014.

[9] G. Zucchelli, M. Marzadori, I. Mounssif, C. Mazzotti, and M. Stefanini, "Coronally advanced flap + connective tissue graft techniques for the treatment of deep gingival recession in the lower incisors. A controlled randomized clinical trial," Journal of Clinical Periodontology, vol. 41, no. 8, pp. 806813, 2014.

[10] A. Brun, N. Moignot, M. L. Colombier, and E. Dursun, "Emerging nanotechnology in non-surgical periodontal therapy in animal models: a systematic review," Nanomaterials, vol. 10, no. 7, p. 1414, 2020.

[11] W. Zakrzewski, M. Dobrzynski, Z. Rybak, M. Szymonowicz, and R. J. Wiglusz, "Selected nanomaterials' application enhanced with the use of stem cells in acceleration of alveolar bone regeneration during augmentation process," Nanomaterials, vol. 10, no. 6, p. 1216, 2020.

[12] G. Zucchelli, M. Mele, M. Stefanini et al., "Predetermination of root coverage," Journal of Periodontology, vol. 81, no. 7, pp. 1019-1026, 2010.

[13] F. Cairo, P. Cortellini, M. Tonetti et al., "Stability of root coverage outcomes at single maxillary gingival recession with loss of interdental attachment: 3-year extension results from a randomized, controlled, clinical trial," Journal of Clinical Periodontology, vol. 42, no. 6, pp. 575-581, 2015.

[14] D. Yaman, K. Demirel, S. Aksu, and C. Basegmez, "Treatment of multiple adjacent Miller class III gingival recessions with a modified tunnel technique: a case series," International Journal of Periodontics and Restorative Dentistry, vol. 35, no. 4, pp. 489-497, 2015.

[15] H. H. Zadeh, "Minimally invasive treatment of maxillary anterior gingival recession defects by vestibular incision subperiosteal tunnel access and platelet-derived growth factor BB," International Journal of Periodontics and Restorative Dentistry, vol. 31, no. 6, pp. 653-660, 2011.

[16] S. R. Dandu and K. R. V. Murthy, "Multiple gingival recession defects treated with coronally advanced flap and either the VISTA technique enhanced with GEM 21S or periosteal pedicle graft: a 9-month clinical study," International Journal of Periodontics and Restorative Dentistry, vol. 36, no. 2, pp. 231-237, 2016.

[17] I. Mounssif, M. Stefanini, C. Mazzotti, M. Marzadori, M. Sangiorgi, and G. Zucchelli, "Esthetic evaluation and patient-centered outcomes in root-coverage procedures," Periodontology 2000, vol. 77, no. 1, pp. 19-53, 2018.

[18] L. Chambrone and D. N. Tatakis, "Periodontal soft tissue root coverage procedures: a systematic review from the AAP Regeneration Workshop," Journal of Periodontology, vol. 86, no. 2-s, pp. S8-S51, 2015.

[19] M. P. Santamaria, F. L. . S. Neves, C. A. Silveira et al., “Connective tissue graft and tunnel or trapezoidal flap for the treatment of single maxillary gingival recessions: a randomized clinical trial," Journal of Clinical Periodontology, vol. 44, no. 5, pp. 540-547, 2017.

[20] T. Thalmair, S. Fickl, and H. Wachtel, "Coverage of multiple mandibular gingival recessions using tunnel technique with connective tissue graft: a prospective case series," International Journal of Periodontics and Restorative Dentistry, vol. 36, no. 6, pp. 859-867, 2016.
[21] S. Raja, T. Kumar, T. Gowda, D. Mehta, and A. Kumar, "Management of multiple gingival recessions with the VISTA technique: an 18-month clinical case series," International Journal of Periodontics and Restorative Dentistry, vol. 38, no. 2, pp. 245-251, 2018.

[22] N. Nizam, O. Bengisu, and S. Sonmez, "Micro- and macrosurgical techniques in the coverage of gingival recession using connective tissue graft: 2 years follow-up," Journal of Esthetic and Restorative Dentistry, vol. 27, no. 2, pp. 71-83, 2015.

[23] J. R. Esteibar, L. A. Zorzano, E. E. Cundín, J. D. Blanco, and J. R. Medina, "Complete root coverage of Miller class III recessions," International Journal of Periodontics and Restorative Dentistry, vol. 31, no. 4, pp. E1-E7, 2011.

[24] S. Aroca, A. Barbieri, M. Clementini, F. Renouard, and M. de Sanctis, "Treatment of class III multiple gingival recessions: prognostic factors for achieving a complete root coverage," Journal of Clinical Periodontology, vol. 45, no. 7, pp. 861$868,2018$. 